\title{
Avaliação dos doentes de cuidados primários Enquadramento conceptual
}

\author{
PEDRO LOPES FERREIRA*
}

\begin{abstract}
Resumo
Este é um artigo dedicado à avaliação da qualidade dos cuidados primários sob a perspectiva dos utentes dos Centros de Saúde. É o primeiro resultado escrito de um projecto internacional iniciado em 1995 e apoiado em parte pela União Europeia, no âmbito do programa BioMed. Em Portugal, tem tido o apoio da Administração Regional de Saúde do Centro.

Opresente artigo é o resultado de uma análise da literatura publicada sobre este assunto e apresenta alguns conceitos relevantes inclúdos numa primeira abordagem do modelo conceptual necessáio à avaliação da qualidade pelos utentes/doentes e por nós utilizado no estudo atrás referido. Para esta análise da literatura existente consultámos publicações periódicas das áreas da medicina geral e familiar, da medicina em geral e de investigação em serviços de saúde, assim como a base de dados bibliográfica Medline.

Uma das conclusões que se pode tirar desta análise é que, pelo menos em Medicina Geral e Familiar, muito pouco progresso tem sido alcançado e muito à ainda a fazer para que a avaliação que os doentes fazem dos cuidados que lhes são prestados seja usada como uma medida de qualidade.
\end{abstract}

Palavras-chave: Satisfaç̃o dos Doentes, Medicina Geral e Familiar, Avaliaccão da Qualidade

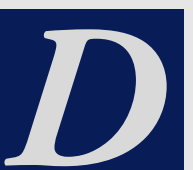

INTRODUCÃO encaradas como fazendo parte dos processos de cuidados médicos. No entanto, em muitos sistemas de saúde, a qualidade ainda é vista apenas sob a perspectiva dos prestadores de cuidados ou dos políticos da saúde e a maioria dos estudos ainda é desenhada de acordo com esses critérios. À perspectiva dos doentes ainda não foi univer-

* Professor Associado Fac. de Economia da Univ. de Coimbra Centro de Estudos e Invest. em Saúde salmente dada uma completa legitimidade para avaliar a qualidade dos cuidados.

Por outro lado, os doentes estão a ter um papel activo cada vez maior nos debates sobre os cuidados de saúde, questionando os cuidados médicos prestados e querendo participar na garantia da qualidade. Esta participação dá-lhes a oportunidade de compreenderem as diferentes opções de tratamento, de fazerem escolhas informadas respeitantes aos cuidados médicos e de se tornarem co-responsáveis pelos resultados dos cuidados. É, por isso, importante que os doentes participem e se envolvam nas actividades de garantia da qualidade dos cuidados médicos. Só assim é possivel aos investigadores dos serviços de saúde - quer sejam prestadores de cuidados, gestores, políticos de saúde ou investigadores em saúde não inseridos no sistema de prestação - iniciar esforços de construção de instrumentos e mecanismos para garantir a qualidade de cuidados de acordo com as expectativas dos doentes.

Os utentes e a sociedade em geral começam já a responsabilizar os prestadores de cuidados sobre os serviços por eles prestados ${ }^{1} \mathrm{e}$ a "pedir-lhes contas" por actos de menos boa qualidade. Ao mesmo tempo, entre os prestadores, os gestores e os agentes de decisão política, assiste-se também a um maior interesse em envolver os doentes nas actividades de garantia da qualidade e em tomar em consideração as avaliações que estes fazem dos cuidados prestados ${ }^{2}$. As tendências re- 
centes têm sido no sentido da utilização de questionários aplicados aos doentes sobre as respectivas experiências com os serviços e com os resultados (ou impactos) dos cuidados prestados. Os estudos da satisfação dos doentes têm, assim, sido usados para documentar as perspectivas dos doentes. ${ }^{3}$

Contudo, pouco conhecimento temos sobre a forma como os utentes avaliam a qualidade dos cuidados $\mathrm{e}$ a que aspectos dão mais relevância. Estas opiniões são importantes para a compreensão do funcionamento dos sistemas de saúde e mantêm normalmente fortes relações empíricas com uma variedade de indicadores dos processos de cuidados e dos resultados desses processos. Um problema que às vezes se põe é que não cobrem todos os aspectos relevantes ou não permitem comparações entre si. De novo segundo Irvine e Irvine, estes estudos estão muitas vezes mais interessados em salvaguardar a imagem dos prestadores de cuidados face aos utentes ou às organizações do que em assinalar problemas reais de qualidade ou em explorar oportunidades para tornar os cuidados de saúde mais dirigidos para os utentes. ${ }^{1}$

Os principais temas de investigação que serão analisados neste artigo de revisão são os seguintes:

- Guais os aspectos da medicina geral e familiar que estão incluídos nas descrições de experiências dos doentes?

- Guais os métodos que têm sido aplicados para obter as opiniões dos doentes?

\section{MÉtodos}

Para responder a estas questões de investigação levámos inicialmen- te a cabo uma análise da literatura existente em 20 publicações periódicas de medicina geral e familiar (British Journal of General Practice, Family Practice, Journal of Family Practice, Revista Portuguesa de Clínica Geral e Scandinavian Journal of Primary Care), de medicina em geral (Acta Médica Portuguesa, British Medical Journal, Journal of Chronic Diseases, Lancet, Medical Care e New England Journal of Medicine) e de investigação em serviços de saúde (Health Services Research, Inquiry, Journal of Health and Social Behavior, Journal of Quality Improvement, Quality in Health Care, Quality Management in Health Care, Quality Review Bulletin, Social Science \& Medicine e Sociology of Health and Illness). De seguida, a pesquisa continuou com base nas referência bibliográficas das publicações encontradas e, por fim, utilizámos a Medline com as palavraschave "satisfação de consumidores" combinada com "qualidade de cuidados".

Temos consciência de algumas limitações desta nossa pesquisa. Em primeiro lugar, apenas analisámos estudos já publicados o que pode ter causado um enviesamento. Em seguida, por questões práticas, apenas percorremos publicações escritas em inglês ou em português. Finalmente, apenas analisámos publicações de cuidados de saúde em geral e de cuidados de saúde primários.

\section{- O Papel do Doente na Garantia de Gualidade}

Se partirmos do pressuposto que o conceito de qualidade é subjectivo, é de esperar que varie consoante seja definido e aplicado por um clİnico, por um doente, por um gestor ou por uma autoridade regional de saúde..$^{4,5,6}$ Qualidade é, talvez, equilibrar todas estas pers-pectivas.
Assim, enquanto Lohr e Harris-Wehling, após terem procedido a uma análise de conteúdo de cerca de uma centena de publicações, definiam qualidade como o aumento de verosimilhança da coerência entre os resultados de saúde desejados e o conhecimento dos profissionais, ${ }^{7}$ Garvin definia, de uma forma geral, o mesmo conceito, agora sob a perspectiva do consumidor, como o grau segundo o qual um conjunto de características de um produto, de um serviço ou de um processo satisfazem as necessidades do consumidor. ${ }^{8}$

Outro ponto de referência a ter em conta sempre que analisamos a qualidade em saúde são os vários aspectos da prestação de cuidados: a avaliação reflecte todos esses aspectos, incluindo os técnicos da aplicação do conhecimento e tecnologias médicas, os interpessoais dos cuidados, os sociais e psicológicos, os éticos da interacção do doente com o prestador de cuidados e a organização de cuidados em termos de acessibilidade, prontidão, continuidade e coordenação.

Concretamente, podemos referir aspectos da clínica geral que variam da informação sobre a doença até ao tempo de espera para uma consulta. Irvine e Irvine, mais uma vez, referem seis aspectos de cuidados primários que consideraram cruciais. São eles os cuidados pessoais, seguros e efectivos, o fácil acesso aos serviços, a escolha dos profissionais de saúde, a continuidade de cuidados, a boa coordenação com outros serviços e os cuidados domiciliários, se necessário. ${ }^{1} \mathrm{O}$ aspecto da autonomia dos doentes foi apresentado por Williamson que o incluiu numa lista também composta por respeito pelos doentes, apoio, informação, controlo, tomada de decisão e escolha. ${ }^{9}$ Um outro aspecto impor- 
tante é o relacionado com as qualidades afectivas do médico. ${ }^{10,11,12}$

Segundo alguns estudos recentes, a satisfação de doentes tem sido considerada uma área de investigação vital na avaliação da qualidade de cuidados prestados pelas instituições de saúde e uma medida de resultados de cuidados de saúde. ${ }^{13,14,15,16,17,18,19,20,21,22,23}$ Psicólogos, médicos, enfermeiros, analistas e políticos de saúde, investigadores de serviços de saúde e analistas da decisão (para mencionar apenas estes) estão cada vez mais a interessar-se não só pelo significado real do aspecto interpessoal dos cuidados, mas também pelo conhecimento que os doentes têm dos aspectos técnicos. ${ }^{5}$ Davies e Ware afirmaram que os consumidores podem fazer avaliações válidas sobre a qualidade dos cuidados prestados, nomeadamente ao identificar as actividades de garantia de qualidade cuja análise de desempenho se pode basear em dados prestados pelos consumidores. ${ }^{24}$ Tem sido provado que os doentes se interessam pela medição e pela melhoria da qualidade $\mathrm{d}^{1,2,13,14,18,19,20,21 \text {. }}$

Um dos argumentos usados contra a utilização de dados dos doentes sobre qualidade de cuidados é que as suas opiniões parecem estar mais relacionadas com as próprias características pessoais dos doentes do que com a qualidade dos serviços prestados. De facto, foram detectadas algumas correlações entre as características e estados de saúde dos doentes e as avaliações que fazem. ${ }^{25,26}$ Contudo, também é verdade que se pode constatar a existência de uma relação estreita entre as características dos cuidados médicos e as características dos doentes e vários estudos longitudinais têm provado que o enviesamento causado pelas características pessoais não é suficiente para invalidar as avaliações feitas pelos consumidores acerca da qualidade dos cuidados a eles prestados. ${ }^{27}$

Algumas pessoas também argumentam que o número das análises e testes administrados aos doentes podem ser considerados indicadores dos níveis de qualidade atribuídos por estes doentes. Contudo, os poucos estudos que se debruçaram sobre a questão da quantidade versus qualidade não apresentaram resultados conclusivos. ${ }^{28,29}$ Outras pessoas manifestaram-se contra a utilização de dados de satisfação de doentes alegando um eventual desacordo entre as opiniões dos médi$\cos$ e as dos doentes relativamente a aspectos técnicos. No entanto, para estudarmos este assunto de uma maneira mais profunda seriam necessários standards e protocolos, o que é dificil, pois os que existem não são universalmente aceites e os prestadores de cuidados ainda não chegaram a um consenso geral sobre o conteúdo desses protocolos. ${ }^{30}$ Talvez devamos ter de nos resignar com a existência de perspectivas diferentes de um mesmo processo de prestação de cuidados. Num estudo recente, Connally e colegas concluíram que as percepções e auto-avaliações dos estados de saúde feitas pelos clínicos são extremamente diferentes dos valores obtidos directamente dos doentes. ${ }^{31}$ Por outro lado, na avaliação da má qualidade, já esperamos um maior consenso entre médicos e doentes. ${ }^{32}$ Estudos têm mostrado que, de facto, pelo menos para problemas de saúde mais frequentes, os consumidores conseguem distinguir os aspectos técnicos considerados bons ou menos bons pelos médicos.

Uma outra crítica desfavorável à utilização de dados de opinião provenientes de doentes e referentes à qualidade de cuidados é que as avaliações destes apenas reflectem a simpatia com que foram recebidos pelos prestadores. ${ }^{33}$ Contudo, também aqui, os resultados das correlações entre as relações interpessoais e os valores das avaliações das componentes técnicas apenas podem ser considerados enviesados se não entrarmos em conta com o que acontece na vida real. Alguns estudos têm mostrado que a manipulação dos aspectos interpessoais possui um efeito insignificante na valorização dos aspectos técnicos. É, pelo menos, possivel afirmar que as características interpessoais da visita ao hospital ou ao médico de família não limitam a capacidade que o utente tem de distinguir niveis de qualidade técnica que são avaliados de uma maneira distinta pelos prestadores. ${ }^{24}$

Finalmente, há quem defenda que existem aspectos respeitantes aos cuidados técnicos que os doentes nem sempre são considerados competentes para julgar, ficando por vezes satisfeitos com cuidados inadequados. Contrariando esta afirmação, podemos afirmar que o doente é o único (perito) capaz de afirmar quais os resultados que se devem procurar, quais os riscos que devem ser aceites em troca de alternativas terapêuticas de melhoria, e a que custo. Deste modo, os doentes dão um contributo único e indispensável para a definição da qualidade dos cuidados prestados e para o estabelecimento dos padrões por que os cuidados devem ser julgados. A experiência dos doentes torna-se, deste modo, necessária à identificação de problemas de qualidade nas áreas da comunicação, da informação, da continuidade de cuidados e da sua coordenação no sistema de saúde. Os doentes podem também fornecer outros dados 
de natureza comportamental ou relativos ao estado funcional de saúde, à qualidade de vida e ao cumprimento de regimes de tratamento.

Também existem argumentos a favor do uso da participação activa dos consumidores. Um primeiro argumento para a utilização dos dados da satisfação dos doentes é que, de facto, estes materializam as suas próprias opiniões na escolha dos médicos e das instituições de prestação de cuidados. Embora isto não seja inteiramente verdadeiro para indivíduos de baixo estrato sócioeconómico, constitui uma decisão fundamental, pois normalmente é o médico de família que escolhe o especialista com quem mantém uma relação continuada, sendo a escolha do hospital dependente da situação geográfica e dos contactos do médico. Além disso, os que classificam com alta pontuação ambos os aspectos, técnico e interpessoal, menos provavelmente mudarão de médico ou de hospital. ${ }^{34,35}$ Os doentes satisfeitos voltam. ${ }^{13,15,20,21,22,36}$

Um outro argumento a favor da utilização das opiniões dos doentes é que estas não são muito dispendiosas. Pouco se tem escrito acerca do custo de inquéritos aos consumidores; no entanto, o custo de obtenção de dados a partir de doentes é provavelmente menor do que a obtenção de dados dos registos clínicos, especialmente se se utilizarem procedimentos postais ou telefóni$\cos .^{37}$ Outras processos mais tradicionais de garantia de qualidade são muito mais dispendiosos do que a obtenção de informação directamente dos doentes.

A informação obtida dos doentes, quer se trate de avaliações, quer se trate de opiniões, pode ser muito exacta. As opiniões relativas aos cuidados ambulatórios parecem ser as mais precisas. ${ }^{38}$ Os doentes da classe média parecem ter mais facilidade em recordar as suas experiências do que os indivíduos de baixo rendimento. ${ }^{39}$ Além disso, as queixas de má prática são muito maiores em doentes insatisfeitos com os seus médicos quando comparadas com a situação de doentes que experimentaram o mesmo tipo de incidente mas estão satisfeitos com a equipa que lhes prestou os cuidados. ${ }^{40}$

Para além disto, tem sido variadíssimas vezes demonstrado que os doentes satisfeitos têm uma maior adesão à terapêutica ${ }^{13,14,16,17,19,23}$. Os doentes podem também ser a única fonte de dados para alguns aspectos interpessoais de cuidados, muitas vezes inexistentes nos registos clínicos. Esta nova fonte de dados, encarando os doentes como peritos na avaliação da sua própria experiência com os sistemas de saúde, pode desempenhar um papel muito importante como complemento da informação já existente. De salientar que o doente é o único que acompanha todo o episódio de prestação de cuidados no sistema de cuidados de saúde. ${ }^{16}$ Isto é particularmente verdadeiro em doentes crónicos. ${ }^{41}$

Um estudo recentemente publicado por Greenfield a colegas evidencia o poder da informação obtida pelos doentes..$^{42}$ Nesta experiência, os doentes foram aleatoriamente distribuídos por dois grupos: no grupo de controlo não houve qualquer intervenção; os doentes no grupo experimental, antes de entrar na sala da consulta, viram um vídeo com instruções para formular melhor as perguntas ao médico. Os médicos para ambos os grupos foram mantidos constantes e os doentes foram estratificados de acordo com o diagnóstico (diabetes ou hipertensão) e com o facto de terem ou não visto o vídeo. Para ambos os diagnósticos, o grupo experimental exerceu um melhor controlo sobre a doença, tendo sido usados parâmetros de natureza fisiológica, não de satisfação. $O$ estudo conclui que as instruções aos doentes podem resultar num melhor controlo sobre o processo da doença e, para além disso, em estudos de follow-up ficou provado que as opiniões dos doentes relativas às interacções doente-médico são tão precisas como os conteúdos dos registos clínicos.

É também importante salientar a distinção que habitualmente se faz entre avaliações (ratings) e opiniões ou descrições de experiências (reports). ${ }^{43}$ As avaliações dos doentes são subjectivas, ao passo que os comentários e opiniões que proferem são objectivos, não implicando isto qualquer ordenação de importância entre ambos os tipos.

Os doentes devem ser participantes na garantia de qualidade dos cuidados médicos. Só ouvindo-os é que é possivel desenvolver a qualidade de cuidados de acordo com as suas expectativas. ${ }^{44}$ Assim, definindo e avaliando a qualidade e fornecendo informação que permita a outros avaliar essa mesma qualidade, os doentes podem contribuir significativamente para a garantia e melhoria da qualidade.

Em resumo, podemos dizer que os doentes podem ser envolvidos nos três tipos de actividades considerados básicos para a melhoria da qualidade. Assim, ao definir o nivel desejado dos cuidados de saúde, os doentes podem ser úteis na criação e no desenvolvimento de padrões, protocolos e iniciativas para uma melhor prestação de cuidados. Ao fazer sugestões, críticas e comentários em inquéritos, em entrevistas ou mantendo diários actualizados, permitem determinar e quantificar a discrepância existente entre o de- 
sejável e o obtido (experimentado), ajudando a documentar esses cuidados. Por último, ao melhorar o diálogo estabelecido com as organizações de saúde e ao transmitir as suas necessidades de cuidados de saúde, estão a contribuir para a melhoria da qualidade desses mesmos cuidados de saúde.

Fundamentalmente, as avaliações dos doentes incidem sobre a forma como os cuidados prestados respondem às suas necessidades e às normas por eles aceites. Também estas avaliações desempenham um papel triplo nos cuidados de saúde. Em primeiro lugar, constituem um juízo sobre a qualidade dos cuidados de saúde, incorporando os valores, as experiências, as expectativas e as percepções dos doentes. Em segundo lugar, são consideradas resultados de satisfação dos doentes em relação aos cuidados, podendo as avaliações positivas ser encaradas como marcas de êxito. Por fim, podem ser vistas como indicadores de detecção de aspectos de cuidados que carecem ser melhorados, "bandeiras vermelhas" que, quando accionadas, significam que algo vai mal e que são necessárias acções correctivas.

Os próprios conceitos de satisfação e do processo que conduz à avaliação pelos doentes são passiveis de várias interpretações. Pascoe definiu avaliação como uma reacção aos aspectos salientes do contexto (estrutura), dos processos e dos resultados da experiência com os cuidados de saúde. ${ }^{45}$ Linder-Pelz acrescentou que estas avaliações podem referir-se a diferentes aspectos numa consulta ou num episódio de cuidados, dizer respeito a um prestador específico, a um centro de saúde ou a um determinado sistema de saúde. ${ }^{46}$ Por outro lado, Hunt precisou que a satisfação não é o grau de agradabilidade da experiência, mas a avaliação de como esta experiência foi agradável, segundo o que deveria ser ou se esperava que fosse $^{47}$; não se trata de uma mera reacção superficial a uma experiência, mas uma atitude geral e estável, também reconhecida, noutros contextos próximos, como qualidade apercebida. ${ }^{48,49}$

Para tornar ainda mais complexo o conceito de avaliação dos cuidados pelos doentes, existe também alguma investigação quantitativa e qualitativa que demostra a instabilidade temporal deste conceito. ${ }^{50,51}$

\section{- A Avaliação dos Doentes de} Cuidados Primários

Embora os estudos sobre a avaliação dos doentes sejam considerados importantes desde já há algum tempo e vários tenham sido descritos nas últimas duas décadas, ainda persiste alguma incerteza e ainda existem algumas perguntas não respondidas neste campo de investigação. Têm, entretanto, aparecido várias recensões da literatura, análises e meta-análises sobre este tópico. ${ }^{15,19,25,26}$

No início dos anos 80, Pascoe elaborou uma recensão de 46 estudos sobre a satisfação dos doentes em cuidados primários. ${ }^{45}$ Concluiu que a satisfação destes doentes desempenha um papel importante na avaliação dos cuidados primários e que pode ser encarada como factor explicativo de comportamento relacionado com a saúde. Encontrou altos valores de satisfação em todos os seus estudos, o que, em sua opinião, revelava a existência de eventuais problemas metodológicos e uma manifesta necessidade de construção de uma tradição de investigação nesta área.

Hall e Dornan analisaram 221 estudos referentes a avaliações feitas pelos doentes sobre os cuidados médicos e concluíram que a literatura existente sobre satisfação era já vasta e diversa, sendo a maioria de origem americana (84\%), ao passo que menos de $10 \%$ correspondiam a estudos realizados na Europa. ${ }^{52,53}$ Os níveis gerais de satisfação encontrados foram altos, embora esta meta-análise também questionasse a validade de algumas das medições. Neste mesmo trabalho, juntamente com percentagens e frequências dos estudos referidos, foi apresentado um índice geral de satisfação incluindo diferentes aspectos de cuidados médicos. Humanidade e qualidade técnica foram atributos classificados no topo, enquanto as posições menos classificadas correspondiam à atenção dos prestadores a outras necessidades dos doentes e à relação destes com o sistema em termos de continuidade de cuidados, acessibilidade, informação prestada e atenção dada aos problemas psicossociais. Além disto, ficou demonstrado que os outros aspectos dos cuidados médicos eram muito poucas vezes medidos. Assim, e de uma maneira geral, os aspectos mais frequentes foram a humanidade demonstrada pelo prestador de cuidados e a informação prestada por ele; os menos frequentemente medidos foram os problemas psicossociais, a continuidade de cuidados e os resultados. É então sugerido pelos autores que esta disparidade de aspectos limita o nosso entendimento sobre o que é a satisfação e que, para além disso, a validade de conteúdo pode ser melhorada pedindo aos doentes que avaliem a importância de cada aspecto. $O$ facto de instrumentos diferentes incluírem aspectos diferentes de satisfação suscitou também - e ainda suscita - questões relativas a 
comparabilidade da satisfação entre estudos. O problema é complexo.

Uma conclusão importante obtida por estes autores foi que os estudos indicavam que os valores de satisfação dos doentes se correlacionavam positivamente com índices obtidos por peritos sobre os cuidados técnicos, mas que era pouco frequente medir este aspecto. Relativamente à maioria destes estudos, fazem notar a necessidade de melhorar a descrição das características dos instrumentos, em termos de validação e testes de fiabilidade, a descrição das características da amostra e dos resultados da satisfação, em termos de valores médios e correlações com outras variáveis. Hall e Dornan sugeriram que uma explicação para o facto dos instrumentos "caseiros" apresentarem pontuações mais altas para a satisfação se deverá encontrar na falta de validade e na utilização de procedimentos metodologicamente pouco correctos.

O estudo de Hall e Dornan revelava por fim a existência de falhas na literatura em relação a alguns domínios e que as características de cuidados médicos eram sempre avaliadas positivamente pelos doentes. Estes resultados são encarados pelos autores como avenidas importantes para investigações futuras, especialmente na avaliação do desempenho do sistema de prestação de cuidados.

Em cuidados hospitalares, Rubin reviu as avaliações feitas pelos doentes e concluiu que a maioria dos estudos não relata testes de fiabilidade das suas medidas e não descreve os procedimentos de validação. ${ }^{54}$ Refere também um eventual enviesamento relativamente às não-respostas, pondo a hipótese de corresponderem a avaliações menos favoráveis sobre a qualidade de cuidados. A diferença entre respondentes e não-respondentes pode confundir comparações entre satisfações dos doentes. Rubin refere ainda que poucos estudos usaram perguntas escritas de uma forma compreensivel e na forma que os doentes usam para descreverem as suas experiências.

Voltando aos cuidados primários, Wensing e colegas analisaram 40 estudos de avaliações feitas por doentes e concluíram que, no campo dos cuidados primários, poucos progressos têm sido conseguidos relativamente ao uso destas opiniões para uma melhoria da qualidade. ${ }^{55}$ Poucas vezes são incluídos nos estudos muitos aspectos de cuidados considerados importantes pelos doentes.

Em termos gerais, os resultados anteriormente descritos são consistentes com as mais recentes conclusões de Hall e Dornan. Os doentes dos clínicos gerais são normalmente questionados em termos da precisão, humanidade, informação prestada e disponibilidade do médico; a autonomia dos doentes, a continuidade de cuidados e a acessibilidade poucas vezes têm sido incluídos nos vários estudos. ${ }^{56}$ No entender destes autores, isto poderá dever-se ao pressuposto de que os doentes são incapazes de avaliar alguns aspectos, argumento de que profundamente discordamos.

Em relação aos métodos aplicados, este estudo sugere a existência de uma grande variedade de métodos, não sendo claro que uns sejam mais válidos e exequíveis do que outros. Por exemplo, não é claro a quantidade de utentes por Centro de Saúde que deverão ser entrevistados para se obterem dados válidos relativos à qualidade dos cuidados prestados. Além disso, várias formas de recolha de dados têm sido usadas, principalmente questionários preenchidos pelos utentes nos Centros de Saúde ou enviados pelo correio. No entanto, a determinação do método mais discriminatório e mais válido em relação aos não-respondentes é uma questão ainda em aberto.

\section{CONCLUSÕES}

Têm sido descritos vários instrumentos internacionalmente validados..$^{22}$ No entanto, são normalmente orientados e desenhados globalmente para o ambiente americano e não são suficientemente específicos para a situação da Medicina Geral e Familiar na Europa.

A conclusão desta breve recensão da literatura é que muitos aspectos considerados de importância para os doentes não são incluídos nos estudos de avaliação dos cuidados primários e que não tem sido dada a necessária atenção às prioridades dos doentes. Há problemas metodológicos relacionados com as validades interna e externa dos estudos de avaliação dos doentes e não há ainda um instrumento universalmente aceite para a avaliação dos doentes em clínica geral. Se a intenção é integrar sistematicamente as avaliações dos doentes em actividades de garantia de qualidade, há a necessidade de se desenvolver um instrumento válido e fiável de medição.

\section{REFERÊNCIAS BIBLIOGRÁFICAS}

1. Irvine $\mathrm{D}$, Irvine $\mathrm{S}$. The practice of quality. Oxford, New York: Radcliffe Medical Press, 1996.

2. Emanuel EJ, Emanuel LL. Four models of the physician-patient relationship. JAMA 1992; 267: 2221-2226.

3. McIver S. Na introduction of ob- 
taining the view of users of health services. London: Kingis Fund Center for Health Services Development, 1991.

4. Berwick D. Health thyself or heal thy system: can doctors help to improve medical care. Qual Health Care 1992; 1: S2-8.

5. Donabedian A. The definition of quality and approaches to its assessment. Explorations in quality assessment and monitoring, volume 1. Ann Arbor, Michigan: Health Administration Press, 1980.

6. Grol R, Wensing M, Jacobs A, Baker $\mathrm{R}$ (eds.). Quality assurance in general practice. The state of the art in Europe. Utrecht: Netherlands Huisartsen Genootschap, 1993.

7. Lohr KN, Harris-Wehling J. Medicare: A strategy for quality assurance. I: A recapitulation of the study and a definition of care. Qual Rev Bull 1991; 17: 6-9.

8. Garvin DA. What does ëproduct qualityí really means? Sloan Manage Rev 1984; 26: 25-53.

9. Williamson C. Whose standards? Consumer and professional standards in health care. Buckingham. Philadelphia: Open University Press, 1992.

10. Ben-Sira $Z$. The function of the professionalis affective behavior in client satisfaction: a revised approach to social interaction theory. J Health Soc Behav 1976; 17: 3-11.

11. Ben-Sira $Z$. The evaluation of medical treatment and competence development of a model of the function of the physicianis affective behavior. Soc Sci Med 1982; 16: 1013-1019.

12. Segall A, Burnett M. Patient evaluation of physician role performance. Soc Sci Med 1980; 14A: 269-278.

13. Aharony L, Strasser S. Patient satisfaction: what we know about and what we still need to explore. Med Care Rev 1993; 50: 49-79.

14. Carr-Hill RA. The measurement of patient satisfaction. J Public Health 1992; 14: 236-249.

15. Cleary PD, McNeil BJ. Patient satisfaction as an indicator of quality care. Inquiry 1988; 25: 25-26.

16. Donabedian A. Quality assurance in health care: the consumeris role. Qual Health Care 1992; 2: 247-251.

17. Lewis JR. Patient views on quality of care in general practice: literature review. Soc Sci Med 1994; 39: 655-670.

18 Linder-Pelz S. Toward a theory of patient satisfaction. Soc Sci Med 1982; 16: 577 -
582.

19. Locker D, Dunt D. Theoretical and methodological issues in sociological studies of consumer satisfaction with medical care. Soc Sci Med 1978; 12: 283-292.

20. Scott A, Smith R. Keeping the customer satisfied: issues in the interpretation and use of patient satisfaction surveys. Int J Qual Health Care 1995; 6: 353359.

21. Vuori H. Patient satisfaction - does it matter? Qual Ass Health Care 1991; 3: 183-189.

22. Ware JE, Snyder MK, Wright WR, Davies AR. Defining and measuring patient satisfaction with medical care. Eval Program Plann 1983; 6: 247-63.

23 Williams B. Patient satisfaction: a valid concept? Soc Sci Med 1994; 38: 509-516.

24. Davies AR, Ware JE. Involving consumers in quality of care assessment. Health Affairs (Spring 1988): 7-33.

25 Lebow JL. Consumer assessments of the quality of the medical care. Med Care 1974; 12: 328-337.

26. Ware JE, Davies-Avery A, Stewart $\mathrm{AL}$. The measurement and meaning of patient satisfaction. Health Med Care Serv Rev 1978; 1: 1-15.

27. Linn LS et al. Consumer values and subsequent satisfaction ratings of physician behavior. Med Care 22, 1984: 804812

28. Davies AR, Ware JE, Brook RH, Peterson JR, Newhouse JP. Consumer acceptance of prepaid and fee-for-service medical care: results from a randomized controlled trial. Health Serv Res 21, 1986 , 429:52.

29. Sox HC, Margulies I, Sox CH. Psychologically mediated effects of diagnostic tests. Annals Int Med 95, 1981: 680:5

30. Eddy, DM. Variations in physician practice: the role of uncertainty. Health Affairs (Summer 1984): 74-89.

31. Connally J. et al. Health perceptions of primary care patients and the influence on health care utilization. Med Care (Suppl. 27, no.3, 1989: S99-109.

32. Ehrlich J, Morehead MA, Trussell $\mathrm{RE}$. The quantity, quality, and costs of medical and hospital care secured by a sample of teamster families in the New York area. New York: Columbia University School of Public Health and Administrative Medicine, 1961.

33. Stiles WB et al. Dimensions of patient and physician roles in medical screening interviews. Soc Sci Med 95, 1981: 680-5.
34. Marquis MS, Davies, AR, Ware, JE. Patient satisfaction and change in medical care provider: a longitudinal study. Med Care 21, 1983: 821-9.

35. Mirowsky J, Ross CE. Patient satisfaction and visiting the doctor: a self-regulating system. Soc Sci Med 17, 1983: 135361.

36. Koehler WF, Fottler MD, Swan JE. Physician-patient satisfaction: equity in the health services encounter. Med Care Rev 1992; 49: 455-484

37. Fowler FJ. Survey research methods. Applied Social Research Methods Series, vol. I. Beverly Hills, CA: Sage, 1984.

38. Linn BS. Burn patientsí evaluations of Emergency Department care. Annals Emerg Med 11, 1982: 255-9.

39. Goldfield N, Pine M, Pine $M$. Measuring and managing health care quality. Procedures, techniques, and protocols. Gaithersburg, Maryland: Aspen, 1992.

40. Vaccarino JM. Malpractice: the problem in perspective. JAMA 238, 1977: 861-3.

41. Memel D. Chronic disease or physical disability? The role of the general practitioner. Br J Gen Pract 1996; 46: 109-113.

42. Greenfield S, Kaplan SH, Ware JE, Yano EM, Frank HJL. Patientsí participation in medical care: effects on blood sugar control and quality of life in diabetes. J Gen Int Med 3, 1988: 448-57.

43. Kaplan S, Ware J. The patientís role in health care and quality assessment. In providing quality care: the challenge to clinicians, eds. Norbert Goldfield and David Nash. Philadelphia: American College of Physicians, 1989: 29.

44. EuroPEP ó European Task Force on Patient Evaluation of General Practice. European study on patient evaluations of general practice care. Research proposal. April 1994.

45. Pascoe GC. Patient satisfaction in primary health care: A literature review and analysis. Eval Program Plann 1983; 6: 185210.

46. Linder-Pelz S. Social psychological determinants of patient satisfaction: a test of five hypotheses. Soc Sci Med 1982; 16: 583-589.

47. Hunt HK. Consumer satisfaction and dissatisfaction: a developing methodology. In: AMA Business Proceedings, 1978: 245-250.

48. Parasuraman A, Zeithaml VA, Berry LL. A conceptual model of service quality and its implications for future research. J Marketing 1985; 49: 41-50. 


\section{Revisões}

49. Zeithaml VA. Consumer perceptions of price, quality, and value: a means-end model and synthesis of evidence. $J$ Marketing 1988; 52: 2-22.

50. Ferreira PL. The use of the multiattribute utility theory to measure the stability of patients' quality judgments. Notas Económicas 1993; 1: 66-77.

51. Fitzpatrick R, Hopkins A. Problems in the conceptual framework of patient satisfaction: an empirical exploration. Sociol Health Illn 1983; 3: 297-311.

52. Hall JA, Dornan MC. Meta-analysis of satisfaction with medical care: description of research domain and analysis of overall satisfaction levels. Soc Sci Med 1988a; 27: 637-44

53. Hall JA, Dornan MC. What patients like about their medical care and how often they are asked: A meta-analysis of the satisfaction literature. Soc Sci Med 1988b; 27: 935-9.

54. Rubin HR. Patient evaluations of hospital care: a review of the literature. Med Care 1990; 28: 3-9.

55. Wensing M, Grol R, Smits A. Quality judgments by patients on general practice care: a literature analysis. Soc Sci Med 1994; 38: 45-53.

56. Hall JA, Dornan MC. Patients sociodemographic characteristics as predictors of satisfaction with medical care: a meta analysis. Soc Sci Med 1990; 30: 811-8.

Recebido em 1/10/97

Aceite para publicação em 11/1/2000

\section{Endereço para correspondência:}

Centro de Estudos e Investigação em Saúde Faculdade de Economia da Universidade de Coimbra

Av. Dias da Silva, 165

3004-512 Coimbra

\section{ASSESSMENT OF PRIMARY CARE BY PATIENTS}

\section{SUMMARY}

This article aims to acess the quality of primary care from the patient's perspective. It is the result of an international project which has started in 1995 and was partially funded by the EU under the Biomed programme. The part of the study performed in Portugal was supported by the Regional Health Authority. This paper is the result of the analysis of the published literature on this topic and pesents some of the maisn concepts involved in the approach of the conceptual model necessary to access quality by patients. The search of the literature included the journals in family medicine, general medicine, research in health services as well as the Medline reference database.

The main conclusion drawn from this study is that much progress has to be done before the patient's assessment can be used as a quality measure of the care delivered.

\section{Key words:}

Patient's Satisfaction, Family Medicine, Quality Assessment 